\title{
EVALUATION OF ROOT RESORPTION FOLLOWING FORMCRESOL PULPOTOMY IN PRIMARY MOLARS
}

\author{
Amr E. Abdel Latif
}

\begin{abstract}
Aim: This study has been implemented in order that the prevalence and pattern of root resorption in mandibular primary molars previously treated with formcresol pulpotomy could be assessed compared with that takes place in apparently sound contralateral teeth.

Subject and methods: The current retrospective cross sectional study has been fulfilled on a sample of 270 periapical radiographs of 118 children (55 females and 63 males) who have been treated at Pediatric Dentistry Department, at the Faculty of Dentistry, Cairo University. All radiographs were screned based on the method presented by Moorrees et al. for purpose of evaluating the process of root resorption. The root resorption process was divided into 5 stages.

Results : It was shown by the results of study that no difference among root resorption between treated and non-treated teeth existed in most of the cases (91 cases). Whereas, accelerated and delayed root resorption were presented by 36 and 8 of cases, consecutively.

Conclusion: It was concluded through investigation in this study that the prevalence of abnormal root resorption in the mandibular primary molars that was previously treated with formcresol pulpotomy was $32.6 \%$.
\end{abstract}

\section{INTRODUCTION}

All roots of primary molars are involved in physiological root resorption of simultaneously. Occlusal trauma, tumor, necrotic pulp, child's age, caries with pulp involvement, presence of pulpotomy or pulpectomy or the absence of restoration are the major predictors of root resorption in primary molars. ${ }^{(1,2)}$. Whereas, other sides, such as separating primary roots and the position as well as the size of follicles of the permanent successor crowns, may be associated factors, too ${ }^{(3,4)}$.

Despite the fact that high success rates have been reported through various studies, pulp therapy has still remained controversial because of fenestrated and tortuous primary root canal morphology, child's immaturity to sufficiently connect their symptoms and making diagnosis overwhelmingly insufficient, in addition to a difficulty in getting a good radiographic view regarding primary root apices. ${ }^{(5,6)}$

* Associate Professor of Pediatric Dentistry and Dental Public Health, Faculty of Dentistry, Cairo University. 
Inflammatory root resorption in primary teeth is a recurrent finding in the pediatric dentists' clinic. Little is known about how pulpotomy of primary teeth influences the occurrence of this type of resorption which is highly frequent in the clinical practice of pediatric dentistry, despite the fact that the prevalence of factors associated with the development of inflammatory resorption in permanent teeth are reported by studies in the literature. The study of this phenomenon is considerably important in the diagnosis and treatment planning in children dental care ${ }^{(7)}$.

The aim of conducting this cross-sectional study was to determine the prevalance and pattern of root resorption in mandibular primary molars that have been previously treated with formcresol pulpotomy matched with that taking place in distinctly sound contralateral teeth.

\section{MATERIALS AND METHODS}

\section{Study design and setting}

The present correlational cross sectional study was accomplished on patients through a random selection from outpatients who were attending postgraduate clinic, at Pediatric Dentistry and Dental Public Health Department, Faculty of Dentistry, Cairo University.

\section{Participants}

Research randomized software (http// ww.randomizer.org/ $/)^{(8)}$ freely available online was followed for purpose of specifying the selected patients per day. The number/codes of the 20 patients /day were randomly selected by software so that they could be included in the study until obtaining total sample size. Having finished ethics committee clearance, patients were enrolled within the study. An information pamphlet in simple nontechnical language and in details was provided beforehand, all patients' parents/guardians included within the study were requested for signing an informed consent. Participates were included in accordance with the following inclusion criteria: six to nine years old children, having the ability for cooperation during radiographic examination, medically free patients, with at least one mandibular molar formerly treated with formcresol pulpotomy along with apparently sound contralateral tooth.

\section{Sample size}

The study sample was enumerated statistically following Raosoft software ${ }^{(9)}$ free online. With reference to steps that were mentioned in the software a hypothesis of 50 percent to obtain the biggest sample size as being recommended. Studying 125 teeth to be able to reject the null hypothesis with probability (power) 0.8 and confidence level $6.39 \%$ were recommended by the final results from this software.

\section{Method}

The current retrospective cross sectional study was carried out on a sample of 270 periapical radiographs of 118 children (55 females and 63 males) treated at Pediatric Dentistry Department, in the Faculty of Dentistry, Cairo University.

A questionnaire was formerly administered to the parents/caregivers of children in order that baseline data on gender, age, dental history and medical history could be acquired. Medical history included the presence/absence concerning general health problems, such as chronic/ systemic diseases. Dental history was followed so as to obtain information related to treatments implemented on the teeth under study.

Concerning radiographic examination, patients having pulpotomized mandibular primary molars, and their contralateral were subject to periapical radiographs, utilizing size 1 periapical films (Eastman Kodak, Rochester, NY, USA). With the aid of a radiographic film holder (Rinn XCP Instruments, Rinn Corporation, Elgin, IL, USA), periapical radiographs were positioned. Only highquality periapical radiographs including sufficient details, good sharpness, consistency and contrast and no distortions displaying the root apices of primary mandibular molars were used. All radiographs were 
screened in accordance with the method introduced by Moorrees et al. ${ }^{(10)}$ for evaluating the root resorption process. The root resorption process was divided into 5 stages which are presented in figure1 with their symbols. Because the investigators were unable to precisely identify the root resorption stage, several teeth were excluded. In both treated and contralateral deciduous molars, the difference between root resorption was investigated by calculating the distribution of the teeth at each stage of root resorption. Two experienced independent investigators examined all radiograph. After 3 months, all 270 radiographs were reevaluated in order that interpreter reliability could be assessed.

\section{Statistical Analysis}

Data were analyzed by adopting the Statistical Package for social science (SPSS. for Windows, version 22, SPSS. Inc., and Chicago, USA). Statistically, data were described in terms of number (n) and percentage (\%) when convenient. A comparison concerning categorical variables was tested by applying Chi-square test, where $P$ value $<0.05$ was considered significant.

\section{RESULTS}

The eventual study sample including 118 children, ranged in age from 6 years 2 months and 8 years 11 months, with a mean age 7 years 3 months. A total of 270 primary molars were studied with the following component: first primary molars $=166$ second primary molars $=104$. After treatment to the day of examination, the elapsed time ranged from 6 to 24 months with a mean of 11.6 months (table 1 ).

\section{Radiographic findings}

Among 135 treated molars, there were a total of 270 radiographs available along with their contralerals. The entire observation was bigger than that of the total number of teeth which existed in the sample, since all the teeth were observed twice (three-month interval) for interpreter reliability.

Table 1 presents distribution of the treated sound teeth and their contralerals among different root resorption stages. The relation between prevalence of root resorption stages in treated and sound teeth was found as being statistically significant $(\mathrm{p}<0.001)$.

Regarding the rate of root resorption, Table 2 presents the number and percentage distribution of treated teeth with regard to their contralateral. When compared with the contralaterals, it was demonstrated by the interpretation of the radiographs that the same stage of root resorption was observed in radiographs of $91(67.4 \%)$ treated teeth, whereas accelerated root resorption was detected in $36(26.7 \%)$ cases. Furthermore; 8 (5.9\%) cases were identified as being delayed root resorption compared to their contralaterals. Statistically, insignificant association was shown between pulpotomized teeth and their contralaterals regarding the rate of root resorption.
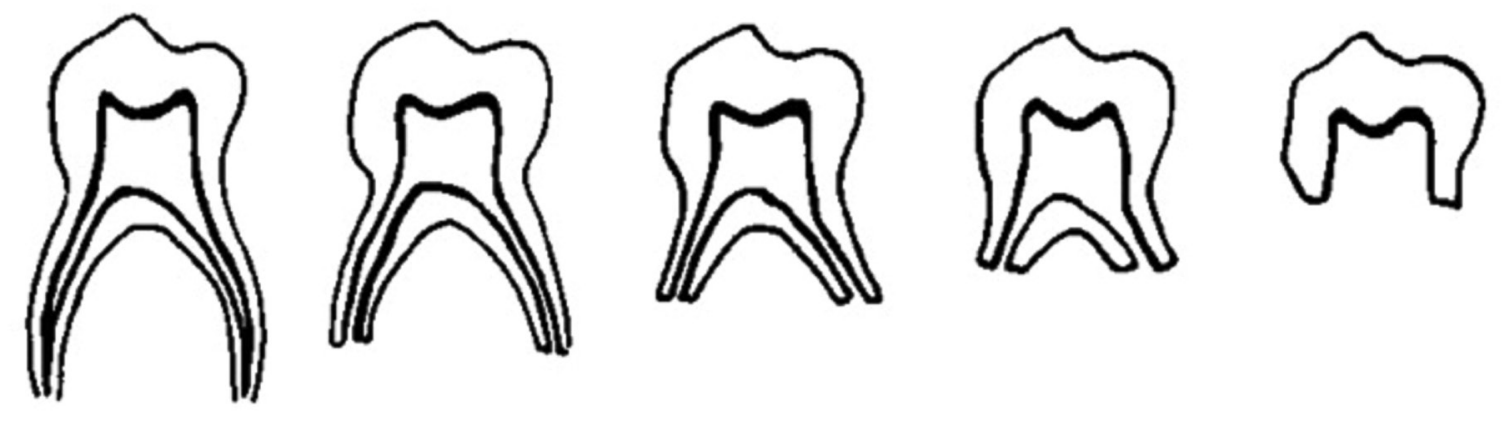

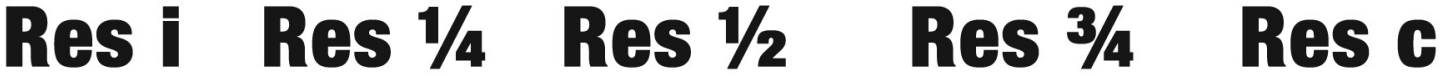

Fig. (1) Stages of root resorption of the deciduous mandibular molars 
TABLE (1) Number and percentage distribution of teeth regarding the five stages of root resorption

\begin{tabular}{|c|c|c|c|c|c|c|c|c|c|c|c|}
\hline & \multicolumn{10}{|c|}{ Root resorption stages } \\
\cline { 2 - 15 } & \multicolumn{2}{|c|}{ Res i } & \multicolumn{2}{|c|}{ Res $1 / 4$} & \multicolumn{2}{|c|}{ Res $1 / 2$} & \multicolumn{2}{|c|}{ Res $3 / 4$} & \multicolumn{2}{c|}{ Res c } & total \\
\hline & No & $\%$ & No & $\%$ & No & $\%$ & No & $\%$ & No & $\%$ & no \\
\hline Treated teeth & 20 & 14.8 & 32 & 23.7 & 57 & 42.2 & 22 & 16.3 & 4 & 3 & 135 \\
\hline contralaterals & 30 & 22.2 & 38 & 28.1 & 46 & 34.1 & 19 & 14.1 & 2 & 1.5 & 135 \\
\hline Total & 50 & 18.5 & 70 & 25.9 & 103 & 38.1 & 41 & 15.2 & 6 & 2.2 & 270 \\
\hline$P$ value & \multicolumn{10}{|c|}{$P<0.001$} \\
\hline
\end{tabular}

TABLE (2) Number and percentage distribution of teeth regarding rate of root resorption

\begin{tabular}{|c|c|c|c|c|c|c|c|c|}
\hline & \multicolumn{2}{|c|}{ No change } & \multicolumn{2}{c|}{$\begin{array}{c}\text { Accelerated root } \\
\text { resorption }\end{array}$} & \multicolumn{2}{c|}{$\begin{array}{c}\text { Delayed root } \\
\text { resorption }\end{array}$} & \multicolumn{2}{c|}{ Total } \\
\hline & no & $\%$ & no & $\%$ & no & $\%$ & no & $\%$ \\
\hline First primary molar & 52 & 38.5 & 26 & 19.3 & 5 & 3.7 & 83 & 61.5 \\
\hline Second primary molar & 39 & 28.9 & 10 & 7.4 & 3 & 2.2 & 52 & 38.5 \\
\hline Total & 91 & 67.4 & 36 & 26.7 & 8 & 5.9 & 135 & 100 \\
\hline P value & \multicolumn{6}{|c|}{0.948} & & \\
\hline
\end{tabular}

\section{DISCUSSION}

Researchers carry on searching for a new pulpotomy material which is harmless to remaining pulp, surrounding tissue where healing of radicular pulp without hindering physiological root resorption will be promoted and be bactericidal ${ }^{(11)}$. For pulpotomy treatment, different materials have been utilized, including formcresol (FC), calcium hydroxide, gluteraldehyde, mineral trioxide aggregate, ferric sulfate and bioactive glass. Among these, FC is the most repeatedly adopted agent in pulpotomy process on primary molars since it is easy to be implemented, with a high success rate, a low cost, as well as being bactericidal.

In the current cross-sectional work, the relationship between the root resorption and pulp therapy (pulpotomy) on the mandibular primary molars was examined. Resorption of primary roots with link to pulp therapy has been investigated formerly by Andrade et al. ${ }^{(2)}$ who documented that abnormal root resorption in $15.3 \%$ and $15.7 \%$ of cases was shown in primary molars formerly treated with pulp therapy when pulpotomy and pulpectomy were conducted, consecutively.

Based on the method of Moorrees et al, ${ }^{(10)}$ root resorption process was evaluated. According to Nikos et al. ${ }^{(12)}$ there is not always a symmetrical reduction of the length of the root in the process of root resorption. Consequently, the degree and the stage of root resorption were frequently judged and estimated by us, not through the symmetrical reduction of the length of the root, but by the decrease in the root original shape.

The percentage distribution of the teeth among rate of root resorption is demonstrated by our findings. Most of the cases (91 cases out of 135) 
in the present study showed no difference in root resorption, between treated and non-treated teeth. Whereas, accelerated and delayed root resorption was presented by $39(27.6 \%)$ and $6(2.2 \%)$ of cases, respectively. Our findings were compatible with those of other investigator's. ${ }^{(8)}$

Children were radiographically tested for purpose of detecting any radiographic proof of changes by using a periapical radiograph size 1 . Periapical radiograph was chosen as it is regarded the best detection technique for periapical changes, periodontal status, osseous defects, any changes in the surrounding structures, and the compatible size of the radiograph aid in getting a positive x-ray experience in children ${ }^{(13)}$. In order to compensate any minor inaccuracies, an interpreter reliability was employed.

Particularly, inflammation that is caused by pulpotomy accelerated the root resorption rate of the deciduous mandibular molars. So, these teeth are expected to exfoliate earlier than their ordinary antimere. The disproportionate root resorption rate, among the treated teeth and their contralaterals was more marked in the first, than in the second deciduous molar (table 1). The interpretation of this phenomenon can be connected to the tooth morphology it seemed easier for inflammation in the first rather than the second deciduous molar to perforate the floor of the pulp chamber and stimulate the osteoclasts. the pulpotomy may begin influencing the rate of root resorption after a bigger time period than that of any other pathological condition although a hastened root resorption process was repeatedly noticed in deciduous molars which underwent pulpotomy. According to the proved information, the accelerated root resorption in primary molars, treated by pulpotomy in the present study, may be due to diagnostic errors made during the pulp condition assessment, or attributed to technical failure during treatment. Based on some studies, the process of root resorption may be accelerated by this procedure ${ }^{(13)}$.
Concerning the connection between time elapsed, after treatment and radiographic evaluation, the minimum time chosen, to be included within the study, was 6 months because the radiographic manifestation usually develops over months ( $>6$ months and up to 1 year). ${ }^{(14)}$

There were no significant differences among first and second primary molars concerning the distribution of the treated teeth were found between stages of root resorption. Furthermore; significant differences between type of tooth and root resorption stage in the contralateral sides $(P=0.021)$.

\section{CONCLUSION}

The prevalence of abnormal root resorption in the mandibular primary molars formerly treated with formcresol pulpotomy that have been investigated in this current study was $32,6 \%$.

\section{REFERENCES}

1. Fuss Z, Tsesis I, Lin S. Root resorption - Diagnosis, classification and treatment choices based on stimulation factors. Dent Traumatology 2003;19:175-82

2. Vieira-Andrade RG, Drumond CL, Alves LP, Marques LS, Ramos-Jorge ML. Inflammatory root resorption in primary molars: Prevalence and associated factors. Braz Oral Res 2012;26:335-40.

3. Haavikko K. Correlation between the root resorption of deciduous teeth and the formation of the corresponding permanent teeth. Proc Finn Dent Soc 1973;69:191-201

4. Harokopakis-Hajishengallis E. Physiologic root resorption in primary teeth: Molecular and histological events. J Oral Sci 2007;49:1-12.

5. Barcelos R, Santos MP, Primo LG, Luiz RR, Maia LC. ZOE paste pulpectomies outcome in primary teeth: A systematic review. J Clin Pediatr Dent 2011;35:241-8.

6. Ozalp N, Saroðlu I, Sönmez H. Evaluation of various root canal filling materials in primary molar pulpectomies: An in vivo study. Am J Dent 2005;18:347-50

7. Rood HD, Waterhouse PJ, Fuks AB, Fayle SA, Moffat MA. UK clinical guidelines in pediatric dentistry: Pulp therapy for primary molars. International Journal of Pediatric Dentistry 1997;7:267-8 
8. http//www.randomizer.org/

9. Raosoft, Inc. www.raosoft.com/samplesize

10. Moorees CF. Fanning EA. Hunt EF. Formation and resorption of three deciduous teeth in children. Am J Phys Anthrop.1963:21;205-213.

11. Sonmez D, Sari S, Cetinbas T. A Comparison of four pulpotomy techniques in primary molars: a long-term followup. J Endod. 2008;34:950-955.

12. Nikos B. Sprios CH,. Nikos M. premature or delayed exfoliatuin of deciduous teeth and root resorption and formation. The Angle Orthodontist 1994;64(1);151-7.

13. Kumar V. Ara K. Kumar HU. Different Radiographic Modalities used for detection of common periodontal and periapical lesion encountered $\backslash$ in routine dental . Oral Hygiene Health.2014;2(5).

14. Havale R, Anegundi R. Indushekar K. Sudha P. Clinical and radiographic evaluation of pulpotomies in primary molars with formcresol, glutaradehyde and ferric sulphate. Oral Health and Dental management.2013;12(1):24-31. 